\title{
Evaluation of the Global Longitudinal Strain in FMF: In Relation with Duration of Illness
}

\author{
(1) Erhan Aygün1, (1) Fatih Mehmet Keleşoğlu2, (1) Gafur Doğdu33, (1) Ömer Kumaş², \\ (1) Rukiye Eker Ömeroğlu3
}

\footnotetext{
1İstanbul University İstanbul Faculty of Medicine, Department of Pediatrics, İstanbul, Turkey

2İstanbul University İstanbul Faculty of Medicine, Department of Pediatric Rheumatology, İstanbul, Turkey

3̇́stanbul University İstanbul Faculty of Medicine, Department of Pediatric Cardiology, İstanbul, Turkey
}

\begin{abstract}
Objectives: The aim of this study is to investigate the effects of Familial Mediterranean Fever (FMF) on cardiac function via speckle tracking echocardiography (ECG) in pediatric FMF patients without cardiac symptoms and to compare speckle tracking ECG results with the conventional echocardiographic outcomes.

Materials and Methods: A total of 50 FMF children aged between 5 and 19 years and 50 healthy children adjusted by age were evaluated for inclusion. Conventional ECG of each participant was performed to investigate mechanical myocardial function. Global longitudinal strain (GLS) measurements were performed using 2D speckle tracking ECG.

Results: The mean duration of illness among FMF patients was $4.6 \pm 3.1$ years. There was no statistically significant relationship between sex and duration of illness ( $>0.05$ ). GLS values measured by two-dimensional speckle tracking between the groups were significantly lower in individuals diagnosed with FMF compared to the control group (-18.0 $\pm 2.0 /-$ $22.5 \pm 2.0 ; \mathrm{p}<0.001)$. The relationship between disease duration and left ventricle-GLS values of individuals diagnosed with FMF was evaluated by spearman correlation analysis. It was observed that left ventricle-GLS values decreased statistically with increasing disease duration.

Conclusion: Speckle tracking ECG can provide the chance to reveal subclinical ventricular dysfunction before cardiac dysfunctions progress in children with FMF.
\end{abstract}

Keywords: Familial Mediterranean Fever, left ventricular function, speckle tracking echocardiography, strain

Address for Correspondence: Erhan Aygün, İstanbul University İstanbul Faculty of Medicine, Department of Pediatrics, İstanbul, Turkey e-mail: dr.erhanaygun@gmail.com ORCID: orcid.org/0000-0002-4495-5824

Received: 10.07.2020 Accepted: 13.11.2020

Cite this article as: Aygün E, Keleşoğlu FM, Doğdu G, Kumaş Ö, Ömeroğlu RE. Evaluation of the Global Longitudinal Strain in FMF: In Relation with Duration of Illness. EJCM 2020;8(4):186-192.

DOI: $10.32596 /$ ejcm.galenos.2020.07.032

${ }^{\circ}$ Copyright 2020 by Heart and Health Foundation of Turkey (TÜSAV) / E Journal of Cardiovascular Medicine published by Galenos Publishing House. 


\section{Introduction}

Familial Mediterranean Fever (FMF) is a hereditary auto inflammatory disease with unknown cause and characterized by attacks of fever, peritonitis, synovitis and pleurisy in the majority of patients, while some may also exhibit skin lesions, vasculitis and amyloidosis ${ }^{(1-5)}$. It has been known for quite some time that the disease spreads into the gastrointestinal system, liver and spleen; however, the involvement of myocardial tissue is a relatively recent discovery ${ }^{(6,7)}$. Although the term cardiomyopathy is not used to define the changes caused by FMF, the disease may mildly affect systolic and diastolic function $^{(8)}$. Conventional echocardiography (ECG) is the most commonly used method in the evaluation of heart involvement and left ventricle function in patients with $\mathrm{FMF}^{(8)}$. Besides, subclinical FMF patients are generally considered normal with conventional ECG and pulsed wave Doppler. Pulsed wave Doppler tissue imaging utilizes both global and regional myocardial function to unveil subclinical cases and examine left ventricular myocardial segments ${ }^{(9,10)}$. Nevertheless, this method cannot discriminate between the movement of myocardial tethering and translational motion and it is affected by the rotational movement of the heart. Therefore, a new technique has been developed: strain and strain rate $\mathrm{ECG}^{(11,12)}$. Strain and strain rate can be determined using speckle tracking ECG (STE). STE emerged as a technique that could determine right and left ventricular function more reliably and comprehensively compared to previous iterations of the ECG technique ${ }^{(13)}$. Being able to singlehandedly determine cardiologic characteristics which would normally require various methods is the most critical advantage. Other distinguishing features of the STE method include angle-independent use, high framerate, less noise-interference, and low inter and intraoperator variance ${ }^{(14)}$.

While the cardiac and left ventricle functions of FMF patients that were measured via tissue Doppler and strain imaging have been previously reported respectively, there are only a few studies which have utilized the STE technique. The aim of this study is to investigate the effects of FMF on cardiac function via STE in pediatric FMF patients without cardiac symptoms and to compare STE results with conventional echocardiographic (CE) outcomes.

\section{Materials and Methods}

All patients and control groups were enrolled in a duration between January 2016 and December 2017 and follow-up provided by the Pediatric Rheumatology Department of İstanbul Medical Faculty. A total of 50 FMF children aged between 5 and 19 years and 50 healthy children adjusted by age were evaluated for inclusion. The diagnosis of familial Mediterranean fever was made considering the Tel-Hashomer criteria ${ }^{(15)}$. When the genetic mutations of the patients were examined, 17 patients were homozygous (MEFV $694 \mathrm{~V}$ ), eight patients were heterozygous, and 24 patients were compound heterozygous. Patients who had fewer than three FMF attacks per year were included. All FMF patients were using colchicine at a dose of 1-1.5 $\mathrm{mg} /$ day, and during echocardiographic evaluation, the disease was in remission period in all individuals. Medical history, disease duration, number of attacks, physical examination, laboratory and echocardiographic findings of FMF patients were recorded. Being over 18 years of age, having non-FMF inflammatory disease, proteinuria, acute attacking, being a smoker, having congenital heart disease/acquired, arrhythmia, moderate or severe heart valve abnormalities, presence of complete bundle branch block, poor echocardiographic evaluation due to poor echogenicity, regional patients with wall motion defect and left ventricular ejection fraction (LVEF) below 55\% were considered as exclusion criteria from the study. LVEF ( $\geq \% 55)$ and Wall motion score (WMSI) $(=1.0)$ were considered as normal left ventricular systolic function. Left ventricular functions were evaluated using conventional ECG method and STE method.

\section{Conventional Echocardiography}

ECG of each participant was performed to investigate mechanical myocardial function. Conventional ECG 
was performed through the parasternal and apical windows by using a Philips IE33 instrument and X5-1 transthoracic probe with the patients in the left lateral position (2-dimensional, M-mode, Doppler ECG). ECG was begun after at least 15 minutes of rest and according to the standard images and techniques in the American and European Cardiovascular Imaging Association guideline $^{(16)}$. All evaluations were performed by a single researcher. M-mode images were obtained from between the mitral valve and papillary muscle on the parasternal long axis. From the parasternal long-axis view of the left ventricule (LV) end-diastolic and endsystolic diameters, interventricular septal and posterior wall thicknesses were expressed in millimeters. We measured LV end-systolic and end-diastolic volumes from the apical 4-chamber view. LVEF was calculated using the modified Simpson biplane method. LV filling was evaluated by pulse wave Doppler from the apical 4-chamber view with the sample volume position at the tips of the mitral valve, and velocities in early (E) and late (A) diastole were recorded, in addition to the calculation of the $\mathrm{E} / \mathrm{A}$ ratio.

\section{Strain Echocardiography Measurements}

Longitudinal global strain (LGS) measurements were performed by using 2D speckle tracking ECG (STE). In these analyses, Philips IE33 and QLAB-CMQ software were used. Measurements were completed using images containing at least 4 cardiac cycles during short apnea period from apical (4 space) windows. The wall motion of each LV segment was visually evaluated on the basis of motion and systolic thickening in a 17-segment model (three segments per wall). Each segment was determined as a conventional four-point scale (1, normokinetic 2, hypokinesia; 3 akinesia; 4, dyskinesia). WMSI was calculated by taking the average value of all segments of each location ${ }^{(17)}$. When the quality was insufficient for analysis, the patient was excluded from the study. The end of diastole was defined in the peak $\mathrm{R}$ wave of the electrocardiogram, while the end of systole was defined as the aortic valve closing time. Endocardial borders were observed within the frame of $2 \mathrm{D}$ images at the end of systole. Manual adjustments were made, if necessary, to ensure correct tracking and to close all LV wall thickness for 2D speckle viewing width.

All participants gave their written informed consent to the study, which was approved by our Institutional Ethical Committee in accordance with the Declaration of Helsinki, from the Clinical Trials Ethics Committee of İstanbul University (decision no and date: 951 and 11.05.2015).

\section{Statistical Analysis}

All analyses were performed on SPSS 19. Normally distributed continuous variables were described as mean \pm standard deviation. Categorical data were described as frequency (percentages). We assessed differences between the groups with the t-test for normally distributed variables and with the chi-square test for categorical data and proportions. The Spearman correlation coefficients were calculated for continuous variables regarding normality. The Kolmogorov-Smirnov test was applied to analyze normality of the data. The Mann-Whitney U test or Student's t-test were applied to compare unpaired samples as needed. $\mathrm{P}<0.05$ values were accepted as statistically significant.

\section{Results}

Fifty of the 100 individuals included in the study were diagnosed as FMF, and their clinical and demographic characteristics are shown in Table 1. There was no difference in age and gender between the groups. While 21 of the 50 patients diagnosed with FMF were male (43\%), 16 of the 50 people in the control group were male $(33 \%)$. The mean age of the individuals diagnosed with FMF was $12.8 \pm 4.2$ years and the mean age of the individuals in the control group was $12.1 \pm 3.6$ years. There was no statistical difference between the groups in terms of body mass index. Similarly, there was no statistical difference between the two groups in heart rate, systolic blood pressure and diastolic blood pressure at admission. When laboratory tests were compared in both groups, creatinine, hemoglobin, hematocrit, leukocyte 
count, sedimentation, C-reactive protein and fibrinogen values were statistically similar. In addition, while all of the individuals diagnosed with FMF were using colchicine, the mean disease duration was determined as 4 (2-6) years (Table 1$)$.

In the conventional echocardiographic examination, individuals who were diagnosed with FMF and control group values were compared in terms of LVEF, left ventricular end diastolic diameter, left ventricular end systolic diameter, interventricular septum thicknessdiastolic (IVSTd), posterior wall thickness (PWT), left atrium (LA), stroke volume, WMSI and diastolic parameter indicators, E wave, A wave, E/A ratio, isovolumic relaxation time (IVRT), and there was no statistical difference between Lateral E', Lateral A' and E'/A' ratio.

Global longitudinal strain (GLS) values measured by two-dimensional speckle tracking between the groups were significantly lower in individuals diagnosed with FMF compared to the control group (-18.0 $2.0 /-22.5 \pm 2.0$; $\mathrm{p}<0.001$ ) (Table 2 / Figure 1).

Table 1. Clinical demographic characteristics of Familial Mediterranean Fever patients and control group

\begin{tabular}{|c|c|c|c|}
\hline Number of patients $(n)$ & $\begin{array}{l}\text { FMF } \\
(n=50)\end{array}$ & $\begin{array}{l}\text { Controls } \\
(n=50)\end{array}$ & $\mathbf{p}$ \\
\hline Age, years & $12.8 \pm 4.2$ & $12.1 \pm 3.6$ & 0.412 \\
\hline Sex (male, \%) & $21(43 \%)$ & $16(33 \%)$ & 0.295 \\
\hline $\mathrm{BMI}, \mathrm{kg} / \mathrm{m}^{2}$ & $19.0 \pm 2.7$ & $20.1 \pm 4.1$ & 0.115 \\
\hline Disease duration (year) & $4(2-6)$ & - & - \\
\hline Colchicine, n (\%) & $50(100 \%)$ & - & - \\
\hline Heart rate (minute) & $85.8 \pm 12.4$ & $82.4 \pm 11.8$ & 0.168 \\
\hline Systolic blood pressure $(\mathrm{mmHg})$ & $106.5 \pm 10.3$ & $108.1 \pm 9.0$ & 0.414 \\
\hline Diastolic blood pressure $(\mathrm{mmHg})$ & $67.5 \pm 7.0$ & $68.5 \pm 5.9$ & 0.474 \\
\hline Creatinine (mg/dL) & $0.7 \pm 0.1$ & $0.8 \pm 0.1$ & 0.681 \\
\hline Hemoglobin (g/dL) & $13.6 \pm 1.5$ & $14.0 \pm 1.7$ & 0.299 \\
\hline Hematocrit (\%) & $40.8 \pm 4.7$ & $42.5 \pm 5.4$ & 0.451 \\
\hline Leukocyte, $\times 10^{3} / \mathrm{mm}^{3}$ & $6.4 \pm 2.2$ & $6.1 \pm 2.1$ & 0.599 \\
\hline Sedimentation, $\mathrm{mm} / \mathrm{h}$ & $9.4 \pm 4.3$ & $8.0 \pm 3.9$ & 0.098 \\
\hline CRP, mg/dL & $5.2 \pm 2.2$ & $4.8 \pm 2.1$ & 0.314 \\
\hline Fibrinogen, gr/L & $2.6 \pm 0.9$ & $2.7 \pm 0.8$ & 0.221 \\
\hline
\end{tabular}

The relationship between disease duration and left ventricular GLS (LV-GLS) values of individuals diagnosed with FMF was evaluated by the spearman correlation analysis. It was observed that LV-GLS values decreased statistically with increasing disease duration $(\mathrm{r}=0.608$ / $\mathrm{p}<0.001$ ) (Figure 2).

\section{Reproducibility}

A total of 15 patients were randomly selected for inter and intra-observer variability analysis. Compatibility of LV-GLS with and between observers and WMSI values was calculated.

The intra-class correlation coefficient for interobserver and intra-observer variability was, respectively: 0.91 [95\% confidence interval (CI), 0.88-0.94] and 0.93 (95\% CI, 0.89-0.96) for LV-GLS; 0.89 (95\% CI, 0.85$0.95)$ and $0.92(95 \% \mathrm{CI}, 0.88-0.95)$ for the WMSI.

\section{Discussion}

In the study, the GLS value of individuals with normal left ventricular systolic function and diagnosed with FMF was analyzed by 2D speckle tracking method:

1. In the GLS examination, strain values were lower in individuals diagnosed with FMF than in the control group. $(-18.0 \pm 2.0 /-22.5 \pm 2.0 ; \mathrm{p}<0.001)$.

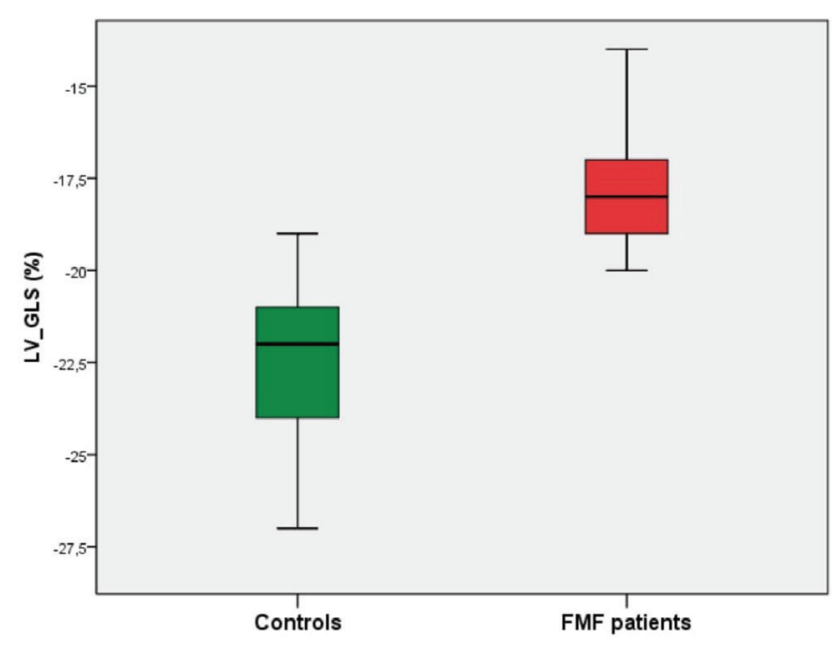

Figure 1. Comparison of LV-GLS values between individuals diagnosed with FMF and the control group

LV-GLS: Left ventricular-global longitudinal strain, FMF: Familial Mediterranean Fever 


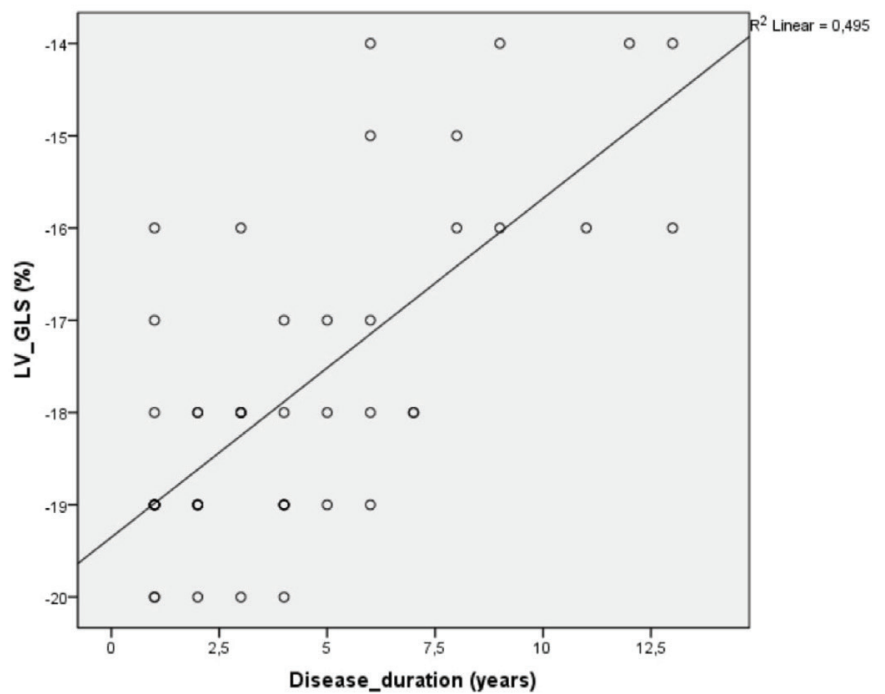

Figure 2. Relationship between disease duration and LV-GLS value in individuals diagnosed with FMF

LV-GLS: Left ventricular-global longitudinal strain, FMF: Familial Mediterranean Fever

Table 2. Features of conventional echocardiographic and twodimensional speckle tracking longitudinal strain parameters of patients

\begin{tabular}{|l|l|l|l|}
\hline Number of patients $(\mathbf{n})$ & $\begin{array}{l}\text { FMF } \\
(\mathbf{n = 5 0})\end{array}$ & $\begin{array}{l}\text { Controls } \\
(\mathbf{n = 5 0 )}\end{array}$ & $\mathbf{p}$ \\
\hline LVEF $(\%)$ & $70.2 \pm 5.5$ & $71.3 \pm 5.9$ & 0.338 \\
\hline LVEDD $(\mathrm{cm})$ & $4.2 \pm 0.5$ & $4.1 \pm 0.5$ & 0.766 \\
\hline LVESD $(\mathrm{cm})$ & $2.4 \pm 0.2$ & $2.5 \pm 0.3$ & 0.399 \\
\hline IVST $(\mathrm{cm})$ & $0.8 \pm 0.2$ & $0.7 \pm 0.2$ & 0.775 \\
\hline PWT $(\mathrm{cm})$ & $0.7 \pm 0.1$ & $0.7 \pm 0.2$ & 0.781 \\
\hline LA $(\mathrm{cm})$ & $2.7 \pm 2.1$ & $2.6 \pm 1.9$ & 0.431 \\
\hline Stroke volume $(\mathrm{mL})$ & $55.2 \pm 19.5$ & $53.4 \pm 18.7$ & 0.573 \\
\hline WMSI & $1.08 \pm 0.12$ & $1.06 \pm 0.14$ & 0.610 \\
\hline E $(\mathrm{cm} / \mathrm{s})$ & $98.2 \pm 17.2$ & $101.5 \pm 16.6$ & 0.492 \\
\hline A $(\mathrm{cm} / \mathrm{s})$ & $58.3 \pm 12.2$ & $56.6 \pm 14.1$ & 0.515 \\
\hline E/A ratio & $1.6 \pm 0.2$ & $1.7 \pm 0.1$ & 0.488 \\
\hline DT $(\mathrm{ms})$ & $120.7 \pm 20.8$ & $118.1 \pm 16.7$ & 0.482 \\
\hline IVRT $(\mathrm{ms})$ & $57.1 \pm 14.3$ & $52.8 \pm 15.8$ & 0.163 \\
\hline Lateral E' $(\mathrm{cm} / \mathrm{s})$ & $13.2 \pm 2.2$ & $12.7 \pm 2.0$ & 0.265 \\
\hline Lateral A'(cm/s) & $7.1 \pm 1.5$ & $6.5 \pm 1.4$ & 0.102 \\
\hline E'/A' & $1.9 \pm 0.4$ & $1.9 \pm 0.5$ & 0.571 \\
\hline LV-GLS $(\%)$ & $-18.0 \pm 2.0$ & $-22.5 \pm 2.0$ & $<0.001$ \\
\hline LVEF & & &
\end{tabular}

LVEF: Left ventricular ejection fraction, LVEDD: Left ventricular enddiastolic diameter, LVESD: left ventricular end-systolic diameter, IVST: Interventricular septum thickness, PWT: posterior wall thickness, LA: Left atrium, WMSI: Wall motion score index, DT: deceleration time, IVRT: Isovolumic relaxation time, LV-GLS: Left ventricular global longitudinal strain, E: Early diastole, A: Late diastole, n: Number
2. A positive and statistically significant relationship was detected between the disease duration and LV-GLS values of individuals diagnosed with FMF ( $\mathrm{r}=0.608$ / $\mathrm{p}<0.001)$.

Some publication reported that ejection fraction (EF) duration was lower in patients compared to controls and they suggested that FMF patients should undergo regular ECG investigations with conventional, strain and strainrate techniques ${ }^{(8,18)}$. Contrastingly, other studies showed that there was no difference regarding EF between adult FMF patients and controls ${ }^{(19,20)}$

Previous studies have reported conflicting results on this matter. In our study, LVEF was not statistically significantly different between the groups. This can be explained due to the fact that the patients were children and the disease periods were short.

In a study, diastolic dysfunction was demonstrated in FMF patients by tissue Doppler imagining by ECG (TDE). In the same study, it was reported that mitral IVRT was prolonged while the mitral lateral annulus $\mathrm{Em}, \mathrm{Am}$, and Em/Am ratio were significantly reduced among FMF patients ${ }^{(20)}$. Kalkan et al. ${ }^{(21)}$, similar to our study, performed strain measurements in addition to TDE in pediatric patients. The authors concluded that systolic strain and strain rate values were damaged in FMF patients, but they could not show the presence of cardiac involvement via the TDE method; the Sm, Em, Am, MPIm and mitral IVRT values were similar for patients and controls. The findings of our study were in contrast with the findings of Tavil et al. ${ }^{(20)}$, but similar to the findings of Kalkan et al. ${ }^{(21)}$.

To summarize, we did not observe any significant difference between the groups regarding TDE findings, while LGS value was found to be lower in the FMF patients.

A statistically significant positive correlation was found between disease duration and LV-GLS values of individuals diagnosed with FMF $(\mathrm{r}=0.608 / \mathrm{p}<0.001)$. 
It shows that the longer it has had FMF disease, the more it has been subclinically affected by heart functions. Studies in adult FMF patients support this ${ }^{(18)}$.

Kalkan et al. ${ }^{(21)}$ also reported that LGS was significantly lower in FMF patients as well as Radial Global Strain, whereas circumferential global strain was similar.

We stated that this might be explained by the fact that the left ventricle endocardium suffered relatively more from adverse effects such as, hypoperfusion, fibrosis, and ischemic changes.

Cardiovascular magnetic resonance (CMR) is a reference standard for the diagnosis of cardiac amyloidosis and is also an excellent tool for risk stratification and disease tracking. It provides accurate measurement of the heart walls and is also useful for the identification of the myocardium. However, considerable experience is required for CMR interpretation. Motion artefacts are generally not present or feasible due to the presence of non-magnetic resonance compatible implantable devices. ECG is crucial for clinical suspicion and initial assessment of cardiac involvement. Amyloid infiltration leads to thickening of the ventricular walls $(12 \mathrm{~mm})$ in a concentric pattern due to the inability of the left ventricular cavity to expand. CMR was not applied in our study ${ }^{(22,23)}$.

After the evaluation of all data, we concluded that reduction in global strain might be an important finding in the early diagnosis of cardiac diseases in pediatric FMF patients.

Our study group was relatively small and consisted of patients with a short disease period. A larger sample group and prospective studies are needed to confirm the results of our study.

\section{Conclusion}

In this study, we evaluated 50 FMF children aged between 5 and 19 years and 50 healthy children with conventional ECG and GLS measurements were performed using 2D speckle tracking ECG. They observed no statistically significant relationship between sex and duration of illness. GLS values measured by twodimensional speckle tracking between the groups were significantly lower in individuals diagnosed with FMF compared to the control group. They conclude speckle tracking ECG can provide the chance to reveal subclinical ventricular dysfunction before cardiac dysfunctions progress in children with FMF.

\section{Ethics}

Ethics Committee Approval: Ethics committee approval was received for this study from the Clinical Trials Ethics Committee of İstanbul University (decision no and date: 951 and 11.05.2015).

Informed Consent: Prospective study. All the patients gave approval.

Peer-review: Internally and externally peer-reviewed.

\section{Authorship Contributions}

Concept: E.A., Design: E.A., F.M.K., G.D., Ö.K., R.E.Ö., Data Collection or Processing: E.A., F.M.K., G.D., Ö.K., Analysis or Interpretation: E.A., F.M.K., G.D., Literature Search: E.A., F.M.K., G.D., Ö.K., R.E.Ö., Writing: E.A., F.M.K.

Conflict of Interest: All the authors had no conflict of interest.

Financial Disclosure: The authors declared that this study received no financial support.

\section{References}

1. Ben-Chetrit E, Touitou I. Familial mediterranean fever in the world. Arthritis Rheum 2009;61:1447-53.

2. Sohar E, Gafni J, Pras M, Heller H. Familial mediterranean fever. A survey of 470 cases and review of the literature. Am J Med 1967;43:227-53.

3. Garcia-Gonzalez A, Weisman MH. The arthritis of familial mediterranean fever. Semin Arthritis Rheum 1992;22:139-50.

4. Lidar M, Doron A, Barzilai A, et al. Erysipelas-like erythema as the presenting feature of familial mediterranean fever. J Eur Acad Dermatol Venereol 2013;27:912-5.

5. Ebrahimi-Fakhari D, Schonland SO, Hegenbart U, et al. Familial mediterranean fever in germany: Clinical presentation and amyloidosis risk. Scand J Rheumatol 2013;42:52-8.

6. Kucuk A, Gezer IA, Ucar R, Karahan AY. Familial mediterranean fever. Acta Medica 2014;57:97-104. 
7. Braun E, Schapira D, Guralnik L, Azzam ZS. Acute vasculitis with multiorgan involvement in a patient with familial mediterranean fever. Am J Med Sci 2003;325:363-4.

8. Ceylan O, Ozgur S, Orun UA, et al. Assessment of left ventricular functions with tissue doppler, strain, and strain rate echocardiography in patients with familial mediterranean fever. Anatol J Cardiol 2015;15:663-8.

9. Nesbitt GC, Mankad S, Oh JK. Strain imaging in echocardiography: Methods and clinical applications. Int J Cardiovasc Imaging 2009;25(Suppl 1):9-22.

10. Triantafyllou KA, Karabinos E, Kalkandi H, Kranidis AI, Babalis D. Clinical implications of the echocardiographic assessment of left ventricular long axis function. Clin Res Cardiol 2009;98:521-32.

11. Hatle L, Sutherland GR. Regional myocardial function a new approach. Eur Heart J 2000;21:1337-57.

12. Shave R, George K, Whyte G, et al. A comparison of doppler, tissue doppler imaging, and strain rate imaging in the assessment of postexercise left ventricular function. Appl Physiol Nutr Metab 2009;34:33-9.

13. Amzulescu MS, Langet H, Saloux E, et al. Improvements of myocardial deformation assessment by three-dimensional speckle-tracking versus two-dimensional speckle-tracking revealed by cardiac magnetic resonance tagging. J Am Soc Echocardiogr 2018;31:1021-33.

14. Petersen JW, Nazir TF, Lee L, Garvan CS, Karimi A. Speckle tracking echocardiography-determined measures of global and regional left ventricular function correlate with functional capacity in patients with and without preserved ejection fraction. Cardiovasc Ultrasound 2013;11:20.

15. Livneh A, Langevitz P, Zemer D, et al. Criteria for the diagnosis of familial mediterranean fever. Arthritis Rheum 1997;40:1879-85.
16. Voigt JU, Pedrizzetti G, Lysyansky P, et al. Definitions for a common standard for $2 \mathrm{~d}$ speckle tracking echocardiography: Consensus document of the eacvi/ase/industry task force to standardize deformation imaging. Eur Heart J Cardiovasc Imaging 2015;16:1-11.

17. Lang RM, Badano LP, Mor-Avi V, et al. Recommendations for cardiac chamber quantification by echocardiography in adults: An update from the american society of echocardiography and the european association of cardiovascular imaging. J Am Soc Echocardiogr 2015;28:1-39.

18. Pamukcu HE DM, Ozisler C, Sunman H, Pamukcu M, Dinc Asarcikli L. Effects of familial mediterranean fever on cardiac functions in adults: A cross-sectional study based on speckle tracking echocardiography. Arch Rheumatol 2019;34:204-10.

19. Canpolat U, Dural M, Aytemir K, et al. Evaluation of various cardiac autonomic indices in patients with familial mediterranean fever on colchicine treatment. Auton Neurosci 2012;167:70-4.

20. Tavil Y, Ureten K, Ozturk MA, et al. The detailed assessment of left and right ventricular functions by tissue doppler imaging in patients with familial mediterranean fever. Clin Rheumatol 2008;27:189-94.

21. Kalkan GY, Bayram NA, Erten S, et al. Evaluation of left ventricle function by strain imaging in patients with familial mediterranean fever. Echocardiography 2010;27:1056-60.

22. Ternacle J, Bodez D, Guellich A, et al. Causes and consequences of longitudinal LV dysfunction assessed by 2D strain echocardiography in cardiac amyloidosis. JACC Cardiovasc Imaging 2016;9:126-38.

23. Nucci EM, Lisi M, Cameli M, et al. The role of 3D and speckle tracking echocardiography in cardiac amyloidosis: a case report. Eur Rev Med Pharmacol Sci 2014;18:74-7. 